Description of the Workshop and of the Methods used to Facilitate Interactions We will present the development of the footnotes checklist. To get hands-on experience the participants will work in large and small groups to: 1) use the checklist on several examples of GRADE evidence profiles and 2) make a judgement about how informative these footnotes are, in particular with guideline panel meetings in mind. The examples will include challenging topics like evidence from single RCT and narrative reviews (no pooled estimates). The outcomes of these exercises will be discussed with the large group and will be used to further improve the checklist.

\section{WS USING A NEW ANALYTIC FRAMEWORK TO CREATE EVIDENCE-BASED COVERAGE GUIDANCE}

V King, A Little, S Vandegriff. Center for Evidence-based Policy, Oregon Health \& Science University, Portland, USA

\section{0:1136/bmjgs-2013-002293.24}

Background A state passed comprehensive health reform legislation in 2009 that directed it to develop a process to translate evidence into coverage guidance to be applied rapidly and uniformly across public and private healthcare payers. A Governorappointed committee managing the state's Medicaid benefit package developed an analytic framework with a decision algorithm to facilitate coverage decisions. The framework is built upon six decision point priorities: sufficiency of evidence, effectiveness of the treatment and availability of alternatives, treatment risk, cost, prevalence of treatment and research feasibility. Objectives/Goal To practice applying decision-making principles and best available evidence to reach coverage decisions.

Target Group, Suggested Audience Policy makers, guideline developers and users.

Description of the Workshop and of the Methods used to Facilitate Interactions A short didactic presentation will present the analytic framework development history. We will discuss alternative priorities that could have been adopted. Participants will then work in facilitated small groups to reach coverage decisions using the framework and algorithm. Each small group will have a summary of the evidence available. The topics will include surgery for femoroacetabular syndrome, carotid endarterectomy and treatment of attention deficit hyperactivity disorder. Facilitators will encourage participants to attempt to reach a coverage decision as if they were a policy-making body and will assist with interpretation of the evidence. The group will identify and consider any potential implementation barriers or considerations and propose management strategies. The groups will share their experience using the framework and the facilitators will present the actual decisions the state committee made.

\section{WS HOW TO USE THE GRADE "EVIDENCE-TO- RECOMMENDATIONS FRAMEWORK" TO DEVELOP GUIDELINE RECOMMENDATIONS FOR THERAPEUTIC INTERVENTIONS}

${ }^{2,3,4,6} \mathrm{H}$ Schunemann, $3,5,6 \mathrm{R}$ Brignardello-Petersen, ${ }^{1,3} \mathrm{~W}$ Chan, ${ }^{3,6,7,8} \mathrm{P}$ Alonso-Coello, ${ }^{1,3,4} \mathrm{M}$ Koster, ${ }^{1,3} \mathrm{C}$ Robbins. ${ }^{1}$ Kaiser Permanente, Pasadena, CA, USA; ${ }^{2} \mathrm{MCMaster}$ University, Hamilton, ON, Canada; ${ }^{3}$ The GRADE Working Group, Hamilton, ON, Canada; ${ }^{4}$ GI-N North America Steering Group, USA; ${ }^{5}$ University of Chile, Santiago, Chile; ${ }^{6}$ DECIDE Project Institute of Biomedical Research Sant Pau Barcelona, Spain, Iberoamerican Cochrane Centre, Barcelona, Spain

10:1136/bmjgs-2013-002293.25
Background Moving from evidence to recommendations in guideline development requires balancing evidence quality with the benefits and harms of therapeutic interventions, patient preferences, and resource and cost considerations. The GRADE Working Group has developed an approach to integrate these factors into development of clinical practice recommendations that is currently further defined in the DECIDE (Developing and Evaluating Communication Strategies to Support Informed Decisions and Practice Based on Evidence) project.

Objectives/Goal To train guideline developers and those working with guideline panels to facilitate the decision-making process for development of recommendations for therapeutic interventions using the GRADE "Evidence-to-Recommendations Framework."

Target Audience Guideline developers, especially those working with guideline panels to develop recommendations for clinical practice.

Description of the Workshop and of the Methods used to Facilitate Interactions An overview of the GRADE "Evidence-to-Recommendations Framework" will be followed by facilitated small group work to develop guideline recommendations. Participants will work together in a simulated guideline panel, and be asked to develop guideline recommendations taking into consideration the quality of evidence from a GRADE evidence summary profile, the balance of benefits vs. harms of an intervention, patient preferences and resource implications. Facilitators will guide the small workgroups through the decision-making process using materials from recent examples of guidelines developed using the "Evidence-to-Recommendations Framework."

\section{WS ELECTRONIC MULTILAYERED GUIDELINE FORMAT: A NOVEL STRUCTURE AND PRESENTATION OF TRUSTWORTHY GUIDELINES AT THE POINT OF CARE}

${ }^{1}$ A Kristiansen, ${ }^{1} \mathrm{P}$ Vandvik, ${ }^{2} \mathrm{P}$ Alonso-Coello, ${ }^{2} \mathrm{D}$ Rigau, ${ }^{1} \mathrm{~L}$ Brandt, ${ }^{3} \mathrm{G}$ Guyatt. ${ }^{1}$ Hospital Innlandet Trust, Gjøvik, Norway; ${ }^{2}$ Iberoamerican Cochrane Centre, Institute of Biomedical Research (IIB Sant Pau), Barcelona, Spain; ${ }^{3}$ Department of Clinical Epidemiology and Biostatistics, McMaster University, Hamilton, Canada

\section{0:1136/bmjqs-2013-002293.26}

Background The DECIDE Project - created by the GRADE Working Group and funded by the European Union - aims at developing and evaluating strategies to improve dissemination and uptake of evidence-based recommendations. Work Package I targets health care professionals and has developed an electronic multilayered guideline format that includes the top layer; consisting of the minimum set of information components deemed necessary for clinicians to act on a recommendation. The first phase of iterative refinements through stakeholder feedback and user testing is completed and we're now initiating the second phase consisting of surveys and randomised trials of alternative formats. Objectives To update participants on the DECIDE project (WP1) and gather feedback on current and alternative guideline formats.

Target Group Guideline developers.

Description The workshop will open with an introduction to the background and progress of the DECIDE project/WP1. Participants will be given a clinical scenario together with relevant examples of guidelines after which they're asked to provide anonymous information on attitudes and perceptions of trustworthy guidelines, the use of GRADE and current presentation formats. Following this they'll be given a systematic review on the same subject and asked to write a draft recommendation in 